\title{
Ustekinumab treats psoriasis by suppressing RORC and T-box but its suppression of GATA restrains its efficacy
}

\author{
Lun-Fei Liu ${ }^{1,2}$, Ji-Su Chen', Ji-Yang Shen ${ }^{3}$, Ting-Ting Dou ${ }^{4}$, Jiong Zhou ${ }^{1}$, Sui-Qing Cai', \\ Min Zheng, ${ }^{1, *}$ \\ ${ }^{1}$ Department of Dermatology, The Second Affiliated Hospital, Zhejiang University School of Medicine, Hangzhou, China, \\ ${ }^{2}$ Department of Dermatology, The Fourth Affiliated Hospital, Zhejiang University School of Medicine, Yiwu, China, \\ ${ }^{3}$ Department of Dermatology, Ningbo Medical Center Lihuili Hospital, Ningbo, China, ${ }^{4}$ Shan Dong Yan Tai Nursing School, \\ Yantai, China
}

\begin{abstract}
Psoriasis is a T-cell mediated disease that involves IL-23/Th17 and IL-12/Th1 axes. Ustekinumab, a fully human monoclonal antibody targeting the $\mathrm{p} 40$ subunit of both IL-12 and IL-23, has proven to be efficient and safe for treating patients with psoriasis. Yet, there have been no reports with human skin/blood samples that would elucidate the molecular mechanisms by which ustekinumab calms psoriasis skin lesions. To investigate the efficacy and molecular pathway (RORC, t-BOX and GATA) of ustekinumab in treating patients with psoriasis skin lesions. A total of 30 patients with psoriasis were randomized into placebo group and treatment group. PASI of each patient was calculated at 0, 12 and 24 weeks post-treatment. The mRNA levels of RORC, t-BOX and GATA in peripheral blood mononuclear cells separated from patients' whole blood were analyzed using qPCR. Decreased mRNA of RORC, t-BOX and GATA were observed after continuous injections, indicating that ustekinumab exerts its effect by interacting with these molecules; while no significant difference in foxp3 mRNA levels were found between placebo group and treatment group.
\end{abstract}

Keywords: Psoriasis. Ustekinumab/efficacy. Biologic agents.

\section{INTRODUCTION}

Psoriasis is a chronic, inflammatory and proliferative skin disease, characterized by impaired differentiation, overactive hyperproliferation of epidermal keratinocytes, disturbed keratinization and aberrant activation of $\mathrm{T}$ lymphocytes. It involves complicated pro-inflammatory cytokine networks, where Th1/Th2 homeostasis, Th17/ Treg balance, IL-23/Th17 axis and IL-12/Th1 axis have shown to have important roles (Deng, Chang, Lu, 2016; Harden, Krueger, Bowcock, 2015). IL-12 and IL-23 have shown to be involved in the development of Th1 and Th17 immune responses in psoriasis (Lee et al., 2004; Yawalkar et al., 2009), and thus have become potential targets for treating patients with psoriasis.

Ustekinumab, a new class of anti-cytokine drugs, is a human monoclonal antibody that targets the $\mathrm{p} 40$ subunit

\footnotetext{
*Correspondence: Min Zheng. Department of Dermatology, The Second Affiliated Hospital, Zhejiang University School of Medicine, Hangzhou, 310009, China. Tel+86571 87784558 / Fax+86571 87215882.E-mail: minz@zju.edu.cn
}

of both IL-12 and IL-23, and thus directly neutralizes their biological activity, and decreases the immune cell activation properties of IL-12 and IL-23. Several clinical trials have demonstrated the superior clinical efficacy and safety of ustekinumab in the treatment of moderate-tosevere psoriasis and psoriatic arthritis (Kavanaugh et al., 2014; Nast et al., 2015; Strober et al., 2016; Tsai et al., 2011) with sustained efficacy after 3 years of treatment (Kimball et al., 2012). Nevertheless, according to PASI75 (PASI: Psoriasis area-and-severity index) its efficiency was lower than in IL-17 pathway blockers, for example, secukinumab (Tan, Griffiths, 2016).

Ustekinumab stimulates peripheral blood monocytes (PBMC) so as to modulate cytokines (Nograles et al., 2008; Reddy et al., 2010). However, to our knowledge, there have been no reports with human skin/blood samples elucidating the pathway by which ustekinumab modulates full blood white cells thus influencing human keratinocytes' cellular processes. There are also no reports on how ustekinumab achieves its efficacy in the treatment of psoriasis and/or presents distinctive properties from other biologic agents. 
Retinoic acid-related orphan receptor $\gamma(\operatorname{ROR} \gamma$, the human form: RORC) is the key transcription factor involved in IL-17-producing cells (Zhang et al., 2015). The inhibition of RORC has shown to be effective in decreasing psoriatic lesions (Rizvi, Chaudhari, Syed, 2015; Takaishi et al., 2017). Furthermore, transcription factor forkhead box P3 (foxp3) is a marked factor of regulatory T cells (Treg) development (Williams, Rudensky, 2007) and its interaction with RORC can inhibit IL-17 (Ichiyama et al., 2008). T-box family of transcription factors is a master determinant of Th1 lineage (Szabo et al., 2000). GATA-3 encodes a protein belonging to the Th2-specific transcription factors, promoting Th2 differentiation (Lantelme et al., 2001) and inducing Th2 cytokine production in an analogous way to T-box (Lee et al., 2000). Dominance of Th1 over Th2 is related with psoriasis (Zhu et al., 2010).

Our aim was to verify our assumption that ustekinumab has longer sustainment because it can interact with RORC, T-box, foxp 3 and GATA. The realtime qPCR was carried out to detect the changes it caused on mRNA levels in patients treated with placebo (P group) and ustekinumab-treated patients (U group).

\section{MATERIAL AND METHODS}

\section{Patients, inclusion and exclusion criteria}

Patients with psoriasis were enrolled in the $2^{\text {nd }}$ Affiliated Hospital, School of Medicine, Zhejiang University, Hangzhou, China. The period of the study was 30 months.

The inclusion criteria were the following: (1) patients with moderate to severe plaque psoriasis; (2) patients between 18-65 years old, diagnosed with plaque psoriasis for at least 6 months before the beginning of the study; (3) patients with PASI $>12$, and skin lesion area takes up $>10 \%$ of total body surface area; (4) patients with no history of biologic agents treatment.

Exclusion standards were: (1) patients with erythrodermic psoriasis or psoriatic arthritis or psoriasis pustulosa or psoriasis guttate; (2) patients with severe and uncontrollable local or systematic acute/chronic infections; (3) patients with active or potential tuberculosis or asthma; (4) patients with malignancy history; (5) patients with other severe systematic disease; (6) patients who were prescribed with drugs or biologic agent; (7) patients who received immunosuppressor within 1 month or (8) psoriasis systematic treatment or phototherapy within 1 month or (9) psoriasis topical agents within 2 weeks.
The study was approved by the Medical Ethics Committee of the $2^{\text {nd }}$ Affiliated Hospital, School of Medicine, Zhejiang University, Hangzhou, China (approval number is 2009-32).

\section{Treatment}

Patients were treated with ustekinumab according to previously described approach (Tsai, Ho, 2011). In the present study, we designed a crossover tests based on the ethics (Figure 1). Patient in the treatment group (U group) were subcutaneously injected with $45 \mathrm{mg}$ ustekinumab at weeks $0,4,16$, and $0.9 \% \mathrm{NaCl}$ at week 12 ; while the placebo group (P group) was subcutaneously injected with $0.9 \% \mathrm{NaCl}$ at weeks 0 and 4 , and $45 \mathrm{mg}$ ustekinumab at weeks 12 and 16.

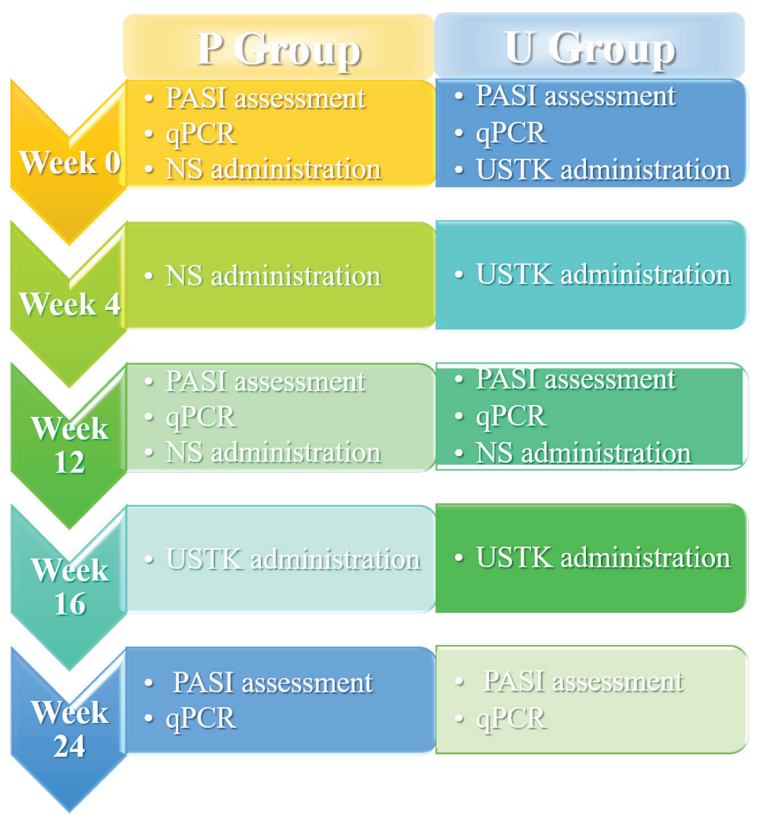

FIGURE 1 - Flowchart of the study. USTK: Ustekinumab. P Group: placebo group. U Group: Ustekinumab group.

\section{Efficacy assessment}

PASI was adopted for the efficacy assessment; when PASI improvement rate reached or exceeded $75 \%$ (PASI-75), the treatment was considered effective. PASI of each patient was calculated at weeks 0,12 and 24, respectively; in weeks 0 and 12, PASI was measured before administration of $\mathrm{NaCl}$ or ustekinumab.

\section{Sample collection}

Whole blood samples were collected at weeks 0,12 and 24 post- treatment. 


\section{Whole blood red blood cell lysis buffer preparation}

10x stock solution was prepared consisting of $89.9 \mathrm{~g}$ $\mathrm{NH}_{4} \mathrm{Cl}, 10.0 \mathrm{~g} \mathrm{KHCO}_{3}, 370.0 \mathrm{mg}$ tetrasodium EDTA, following $\mathrm{pH}$ adjustment to 7.3. The solution was then dissolved in 1 liter of pure water.

\section{RNA extraction and real-time quantitive PCR}

Red blood cells in whole blood samples were dissolved by whole blood red blood cell lysis buffer. Then total RNA was isolated from patients' peripheral leukocytes using invitrogen ${ }^{\mathrm{TM}}$ Trizol $^{\mathrm{TM}}$. cDNA was prepared using Superscript II Reverse Transcriptase (Invitrogen). Gene expression was determined using SYBR green PCR mix (Roche) and $10 \mathrm{ng}$ template. Real-time PCR analysis was performed using ABI StepOne Plus instrument under the following amplification conditions: $5 \mathrm{~s}$ at $95^{\circ} \mathrm{C}$, followed by 45 cycles of $30 \mathrm{~s}$ at $60^{\circ} \mathrm{C}(\mathrm{RORC}) ; 5 \mathrm{~s}$ at $95^{\circ} \mathrm{C}$, followed by 45 cycles of $30 \mathrm{~s}$ at $60^{\circ} \mathrm{C}(\mathrm{FOXP}) ; 5 \mathrm{~s}$ at $95^{\circ} \mathrm{C}$, followed by 45 cycles of $30 \mathrm{~s}$ at $60^{\circ} \mathrm{C}$ (T-box 21$) ; 5 \mathrm{~s}$ at $95{ }^{\circ} \mathrm{C}$, followed by 45 cycles of $30 \mathrm{~s}$ at $60^{\circ} \mathrm{C}$ (GATA). Primers for RORC (5' -CAATGGAAGTGGTGCTGGTTAG-3', 5' - GGGA GT G GGA GAA G TCAAAGAT-3' ), FOXP3 (5'-ATTCCCAGAGTTCCTCCACAAC-3', 5' - A T T G A G T G T C C G C T G C T T C T - 3'), T-box (5'-GCCCCTTCTCCTTTTGATAA-3', 5' '-CGGTGTCCTCCAACCTAATAAC-3') and GATA ( 5'-AGACCACCACAACCACACTCT-3', 5'-GATGCCTTCCTTCTTCATAGTCA-3') were designed using Beacon designer (Premier Software). Transcript expression was normalized to the GAPDH house-keeping gene and represented as either $2^{-\Delta \Delta C}{ }_{T}\left(2^{-\Delta \Delta C}{ }_{T}=2^{-\Delta C}{ }_{T}\right.$ (gene of interest/average $\left[2-\Delta \mathrm{C}{ }_{\mathrm{T}}\right.$ (house-keeping gene) $]$ ) in the case of $\mathrm{p} 35$ or $\mathrm{p} 40$ expression.

\section{Statistics and graph making}

Differences between every two groups were evaluated with unpaired two-tail $t$-test. PASI75 rates (percentages of PASI improvement rate reaching or exceeding $75 \%$ of $\mathrm{P}$ and $\mathrm{U}$ groups, PASI75\%) were compared between $\mathrm{P}$ and $\mathrm{U}$ groups using kappa test. PASI improvement rate (W12vsW0 or $\mathrm{W} 24 \mathrm{vsW0})=[\mathrm{PASI}($ Week0)-PASI(Week12 or Week24)]/ PASI(Week0). ${ }^{*} \mathrm{p}<0.05, * * \mathrm{p}<0.005, * * * \mathrm{p}<0.0001$. Data analysis and graphs were performed using Prism software (GraphPad software, Inc.). In all the graphs, • stands for $\mathrm{P}$ group, and $\boldsymbol{\Delta}$ stands for $\mathrm{U}$ group.

\section{RESULTS}

A total of 30 patients with psoriasis were enrolled (age: 18-60 years old; 7 females and 23 males). Initial PASI ranged from 12.2 to 55.6. The average PASI was 22.86. The standard error of mean (SEM) of PASI was 1.96. Patients were double-blindly and randomly divided into placebo group (P group, $\mathrm{n}=16$, average $\mathrm{PASI}=23.06$, $\mathrm{SEM}$ of PASI $=$ 3.40) and ustekinumab-treated group (U group, $n=14$, average $\mathrm{PASI}=22.64$, SEM of PASI=1.80). One patient did not sign the informal consent for the mRNA level test, therefore his mRNA data were excluded from the study.

\section{Maximum drug efficacy can be obtained within 2 injections of ustekinumab}

To demonstrate the clinical efficacy of ustekinumab, we investigated the intergroup and intragroup comparisons of individual's PASI at 3 different time-points (Figure 2). Briefly, a significant decrease in PASI was observed in $\mathrm{U}$ group at week 12 compared to $\mathrm{P}$ group. In addition, in the U group, PASI decreased significantly at weeks 12 and 24, compared to week 0 ; nevertheless, no significant difference was found between weeks 12 and 24. Besides, in the P group, PASI decreased significantly at week 24 compared to week 0 .

With the reference to PASI-75 rate, higher PASI-75 rate was found in the $\mathrm{U}$ group compared to P group, and

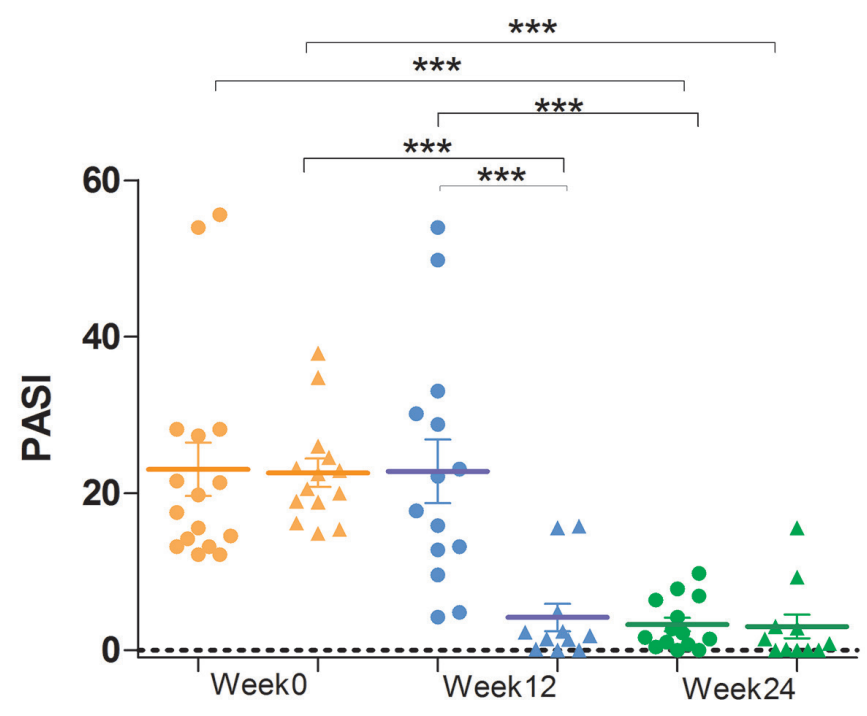

FIGURE 2 - Intragroup and intergroup comparisons of PASI in different timepoints. There are highly significant differences between $\mathrm{P}$ group and $\mathrm{U}$ group at Week 12. There are significant differences in $\mathrm{P}$ group between week 0 and week 24, in U group between weeks 0 and 12, 24 respectively. • stands for P group, and $\boldsymbol{\Delta}$ stands for $\mathrm{U}$ group. 
TABLE I - Kappa tests of PASI of P group and U group, comparing week 12 to week 0, week 24 to week 0 respectively

\begin{tabular}{lcccc}
\hline & \multicolumn{2}{c}{ W12 vs. W0 } & \multicolumn{2}{c}{ W24 vs. W0 } \\
\cline { 2 - 5 } & PASI-75 (n) & $\begin{array}{c}\text { PASI improvement } \\
\text { rate } \leq \mathbf{7 5 \%} \text { (n) }\end{array}$ & PASI-75 (n) & $\begin{array}{c}\text { PASI improvement } \\
\text { rate } \leq \mathbf{7 5 \%} \text { (n) }\end{array}$ \\
\hline P group & 2 & 14 & 13 & 3 \\
U group & 10 & 4 & 12 & 2 \\
P value & - & $0.001^{* *}$ & - & 0.74 \\
\hline
\end{tabular}

this was also verified by kappa test (Table I); significant differences were found at week 12 compared to week 0 $(\mathrm{p}=0.001013)$, while no significance was detected between week 24 and week $0(p=0.74)$, indicating no significant improvement in PASI-75 rate at week 24.

To sum up, the results indicated that ustekinumab is effective in treating psoriasis skin lesions; ustekinumab can reach the maximum efficiency after 2 injections, and then its treatment effect remains constant overtime.
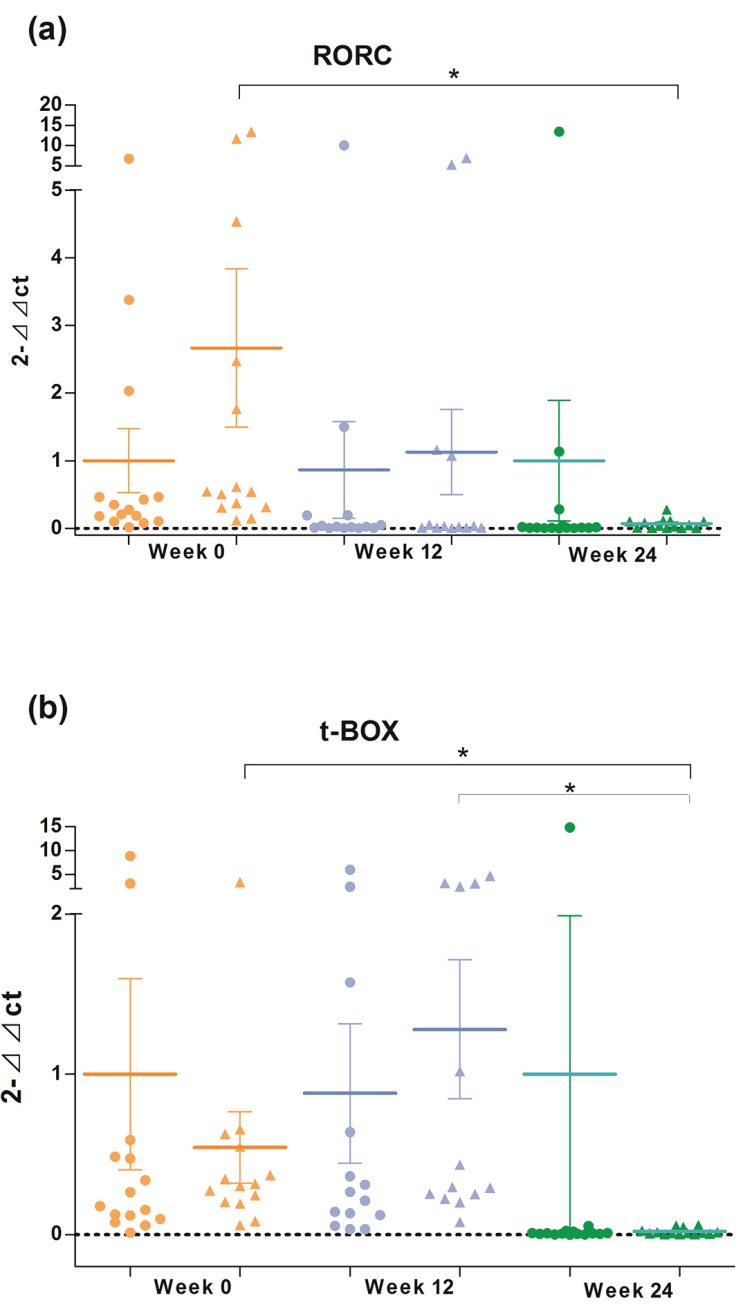

\section{Multiple injections of ustekinumab suppresses RORC, t-BOX, GATA but not foxp3 mRNA}

With reference to RORC mRNA, a significant difference between week 0 and week 24 was found in group $\mathrm{U}$ (Figure 3a), while in $\mathrm{P}$ group there were no significant differences at different time-points, and between $\mathrm{U}$ group and $\mathrm{P}$ group. Furthermore, a significant difference in t-BOX and GATA mRNA levels between

(c)
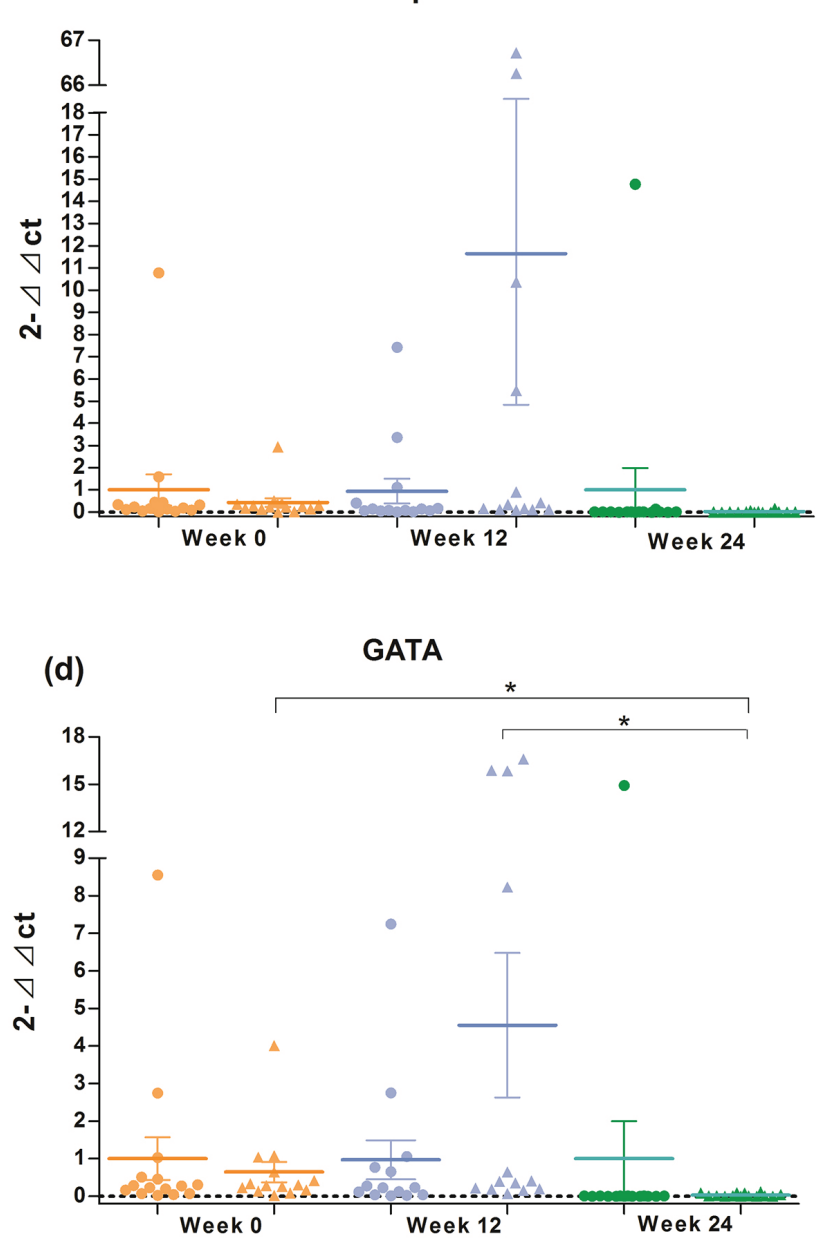

FIGURE 3 - Intergroup and intragroup comparisons of RORC (a), t-BOX (b), foxp3 (c) and GATA (d) mRNA levels at weeks 0, 12 and 24. stands for P group, and $\boldsymbol{\Delta}$ stands for U group. 
week 24 and weeks 0 or 12 were found in group U (Figure $\mathbf{3 b}$ and $\mathbf{3 d}$, respectively). Hence, this raises a question if the expressions of GATA and t-BOX are parallel one to another. In addition, no significant difference in foxp3 mRNA levels were found between groups (Figure 3c).

To sum up, our data suggested that ustekinumab suppresses RORC, t-BOX and GATA. In addition, the maximum suppression effect of RORC can be achieved after 3 injections, while the maximum suppression effect of both t-BOX and GATA can be achieved after 2 injections. Also, it seems that ustekinumab has no effect on foxp3 expression.

\section{DISCUSSION}

Great progress has been made in declaring different roles of different $\mathrm{T}$ cell subsets. Among these cell types, T helper 17 (Th17) and other IL-17-producing cells, notably $\gamma \delta \mathrm{T}$ cells and CD $8+\mathrm{T}$ cells, have been in the center of attention. IL-23/Th17 axis mainly controls the proinflammatory loop in psoriatic plaques (Diani, Altomare, Reali, 2016; Kryczek et al., 2008). Based on our PASI assessment, there were significant differences between $\mathrm{P}$ group and $\mathrm{U}$ group at week 12 post-treatment. There were significant differences in $\mathrm{P}$ group between weeks 0 and 24, in $U$ group between weeks 0,12 , and 24 respectively. These results indicated that two injections of ustekinumab were sufficient for maximal efficacy. According to the instructions, in case of moderate to severe plaque psoriasis and active psoriatic arthritis, the recommended dose of ustekinumab is 45 $\mathrm{mg}$ administered at week(s) 0 and 4, and then every 12 weeks. Therefore, we hypothesized that the third and following injections might intensify ustekinumab's effect or prolong its efficacy.

RORC is the key transcription factor involved in the production and regulation of IL-17 from Th17, $\gamma \delta \mathrm{T}$ cells, innate lymphoid (ILCs), lymphoid tissue inducer (LTi) cells (Fauber, Magnuson, 2014; Isono, Fujita-Sato, Ito, 2014; Yang et al., 2014; Zhang, Luo, 2015) and IL-22 and IL-23 from Th17 (Smith et al., 2016). Various antibodies, both upstream and downstream of RORC, specifically anti-IL-12p40 and anti-IL-17A agents have shown to be efficacious in the treatment of psoriasis (Rizvi, Chaudhari, 2015). Takaishi et al. (2017) have described attenuation of psoriasis-like lesions in two independent psoriasis mouse models after oral administration of ROR $\gamma \mathrm{t}$ (a specific transcript of the RORC gene) antagonist and suggested that this effect was based on neutralization of IL-17producing cells. Our data showed that ustekinumab has an RORC-suppressing effect during long-term use, which is helpful in improving psoriatic skin lesions, and probably associated with its anti-IL12/23 effect.

Foxp3 is one of the most specific markers used to identify Regulatory T Cell (Tregs). Overexpression of Foxp3 is required to maintain function and lineage identity of mature peripheral Tregs (Williams and Rudensky, 2007). Upregulated Foxp3 initially binds to ROR $\gamma t$, thereby reducing IL-17A expression and inhibiting ROR $\gamma$ t-mediated IL-17A promoter activity (Ichiyama, Yoshida, 2008). Etanercept, a TNF inhibitor, can induce transcriptional levels of Foxp3, STAT3 and STAT4 mRNA in responding patients with psoriasis (Quaglino et al., 2011). Adalimumab, also a TNF-inhibiting medication expands functional Foxp3+ Tregs via binding monocyte membrane TNF (Nguyen et al., 2016). Accordingly, foxp3 is upregulated by the neutralization of TNF. Our results indicated that ustekinumab didn't suppress or enhance foxp3 mRNA levels, at least not within 3 injections. Probably, ustekinumab decreases psoriatic skin lesions independently from TNF pathway. On the other hand, it can be used as a substitute for anti-TNF agents in patients not responding to anti-TNF agents, since psoriasis has multiple pathogenic mechanisms.

As the upstream of the IL-17 drives the proinflammatory loop, Th1 produces interferon- $\gamma($ IFN $-\gamma)$ favoring the recruitment and expansion of IL-17-producing cells (Diani, Altomare, 2016; Kryczek, Bruce, 2008). T-box family of transcription factors is a main determinant of Th1 lineage (Szabo, Kim, 2000). Memory CD4+ T cells express a high level of T-box. Pre-mobilization of T-box into nuclei might have more important roles at early time points for rapid responses of memory T cells (Yu et al., 2014). Expression of the human T-box correlates with IFN- $\gamma$ expression in Th1 and natural killer cells (Yu, Zhang, 2014), suggesting that this gene is initiating Th1 lineage development from naive Th precursor cells. T-box sustains the effector function of CD8+ T cells through regulation of CD8+ T cell proliferation, suppressed expression of inhibitory receptors such as PD-1, and promotion of IFN- $\gamma$ and perforin secretion (Lazarevic, Glimcher, 2011). Our data demonstrated that ustekinumab can inhibit T-box expression.-Combining current discoveries, it needs further investigation whether ustekinumab can affect nuclei-located T-box.

GATA-3 encodes a protein that belongs to the Th2-specific transcription factors, promoting Th2 differentiation (Lantelme et al., 2001) and inducing Th2 cytokine production in an analogous way to T-box (Lee, Takemoto, 2000). Regulation of Th1/Th2 balance can be mediated by the level of T-box and GATA-3 (Park et al., 2009). The expression of GATA is, however, markedly 
up-regulated in cells differentiating along the Th2 lineage, and is down-regulated in cells differentiating along the Th1 pathway (Frisullo et al., 2006). Compared to healthy controls, a significantly higher expression of T-box mRNA and lower expression of GATA-3 mRNA were tested in PBMCs of psoriatic patients, and consequently, a much higher T-box/GATA-3 ratio was found in patients than in controls (Zhu et al., 2010). The high T-box and low GATA-3 expression in PBMCs and lesioned skin of psoriatic patients indicates a skew towards the Th1 pathway of T-helper cell activation. T-box and GATA-3 might be regulator genes in psoriasis via the Th1/Th2 balance. Furthermore, our data showed that ustekinumab suppressed GATA expression. From this perspective, it antagonizes the treatment of psoriasis.

To sum up, from the aspect of molecular mechanism, ustekinumab can be seen as a double-edged sword in psoriasis treatment. On the positive side, it suppresses the expression of RORC and T-box. On the negative side, it suppresses the expression of GATA and has no impact on foxp3. The effectiveness of Ustekinumab independent of TNF pathway reveals its potential effect for patients not responding to anti-TNF agents. Screening out patients responding to ustekinumab is the key to making best of the equilibrium between positive and negative sides and to obtaining the most ideal treatment outcome. Future randomized clinical trials are necessary to verify these findings.

\section{DISCLOSURE} interest.

The authors declare that they have no conflicts of

\section{ACKNOWLEDGMENTS}

This research was supported by the National Natural Science Foundation of China (NSFC) (81402594) and Zhejiang Provincial Health Technology Planning Project (2015KYB166).

\section{REFERENCES}

Deng Y, Chang C, Lu Q. The inflammatory response in psoriasis: a comprehensive review. Clin Rev Allergy Immunol. 2016;50(3):377-89.

Diani M, Altomare G, Reali E. T Helper Cell Subsets in Clinical Manifestations of Psoriasis. J immunol Res. 2016;2016:7692024.
Fauber BP, Magnuson S. Modulators of the nuclear receptor retinoic acid receptor-related orphan receptor-gamma (RORgamma or RORc). J Med Chem. 2014;57(14):5871-92.

Frisullo G, Angelucci F, Caggiula M, Nociti V, Iorio R, Patanella AK, et al. pSTAT1, pSTAT3, and T-bet expression in peripheral blood mononuclear cells from relapsing-remitting multiple sclerosis patients correlates with disease activity. J Neurosci Res. 2006;84(5):1027-36.

Harden JL, Krueger JG, Bowcock AM. The immunogenetics of Psoriasis: A comprehensive review. J Autoimmun. 2015;64:6673.

Ichiyama K, Yoshida H, Wakabayashi Y, Chinen T, Saeki K, Nakaya M, et al. Foxp3 inhibits RORgammat-mediated IL-17A mRNA transcription through direct interaction with RORgammat. J Biol Chem. 2008;283(25):17003-8.

Isono F, Fujita-Sato S, Ito S. Inhibiting RORgammat/Th17 axis for autoimmune disorders. Drug Discovery Today. 2014;19(8):1205-11.

Kavanaugh A, Ritchlin C, Rahman P, Puig L, Gottlieb AB, Li S, et al. Ustekinumab, an anti-IL-12/23 p40 monoclonal antibody, inhibits radiographic progression in patients with active psoriatic arthritis: results of an integrated analysis of radiographic data from the phase 3, multicentre, randomised, double-blind, placebo-controlled PSUMMIT-1 and PSUMMIT-2 trials. Annals Rheumatic Diseases. 2014;73(6):1000-6.

Kimball AB, Gordon KB, Fakharzadeh S, Yeilding N, Szapary PO, Schenkel B, et al. Long-term efficacy of ustekinumab in patients with moderate-to-severe psoriasis: results from the PHOENIX 1 trial through up to 3 years. Brit J Dermatol. 2012;166(4):861-72.

Kryczek I, Bruce AT, Gudjonsson JE, Johnston A, Aphale A, Vatan L, et al. Induction of IL-17+ T cell trafficking and development by IFN-gamma: mechanism and pathological relevance in psoriasis. J Immunol. 2008;181(7):4733-41.

Lantelme E, Mantovani S, Palermo B, Campanelli R, Sallusto F, Giachino C. Kinetics of GATA-3 gene expression in early polarizing and committed human $\mathrm{T}$ cells. Immunology. 2001;102(2):123-30.

Lazarevic V, Glimcher LH. T-bet in disease. Nat Immunol. 2011;12(7):597-606. 
Lee E, Trepicchio WL, Oestreicher JL, Pittman D, Wang F, Chamian F, et al. Increased expression of interleukin 23 p19 and p40 in lesional skin of patients with psoriasis vulgaris. J Exp Med. 2004;199(1):125-30.

Lee HJ, Takemoto N, Kurata H, Kamogawa Y, Miyatake S, O'Garra A, et al. GATA-3 induces T helper cell type 2 (Th2) cytokine expression and chromatin remodeling in committed Th1 cells. J Exp Med. 2000;192(1):105-15.

Nast A, Jacobs A, Rosumeck S, Werner RN. Efficacy and Safety of systemic long-term treatments for moderate-to-severe psoriasis: a systematic review and meta-analysis. J Invest Dermatol. 2015;135(11):2641-8.

Nguyen QD, Merrill PT, Jaffe GJ, Dick AD, Kurup SK, Sheppard J, et al. Adalimumab for prevention of uveitic flare in patients with inactive non-infectious uveitis controlled by corticosteroids (VISUAL II): a multicentre, double-masked, randomised, placebo-controlled phase 3 trial. Lancet. 2016;388(10050):1183-92.

Nograles KE, Zaba LC, Guttman-Yassky E, Fuentes-Duculan J, Suarez-Farinas M, Cardinale I, et al. Th17 cytokines interleukin (IL)-17 and IL-22 modulate distinct inflammatory and keratinocyte-response pathways. Brit J Dermatol. 2008;159(5):1092-102.

Park HJ, Lee CM, Jung ID, Lee JS, Jeong YI, Chang JH, et al. Quercetin regulates Th1/Th2 balance in a murine model of asthma. Int Immunopharmacol. 2009;9(3):261-7.

Quaglino P, Bergallo M, Ponti R, Barberio E, Cicchelli S, Buffa E, et al. Th1, Th2, Th17 and regulatory T cell pattern in psoriatic patients: modulation of cytokines and gene targets induced by etanercept treatment and correlation with clinical response. Dermatology. 2011;223(1):57-67.

Reddy M, Torres G, McCormick T, Marano C, Cooper K, Yeilding N, et al. Positive treatment effects of ustekinumab in psoriasis: analysis of lesional and systemic parameters. $\mathrm{J}$ Dermatol. 2010;37(5):413-25.

Rizvi S, Chaudhari K, Syed BA. The psoriasis drugs market. Nat Rev Drug Discov. 2015;14(11):745-6.

Smith SH, Peredo CE, Takeda Y, Bui T, Neil J, Rickard D, et al. Development of a Topical treatment for psoriasis targeting RORgamma: from bench to skin. PloS One. 2016;11(2):e0147979.
Strober BE, Bissonnette R, Fiorentino D, Kimball AB, Naldi L, Shear NH, et al. Comparative effectiveness of biologic agents for the treatment of psoriasis in a real-world setting: Results from a large, prospective, observational study (Psoriasis Longitudinal Assessment and Registry [PSOLAR]). J Am Acad Dermatol. 2016;74(5):851-61 e4.

Szabo SJ, Kim ST, Costa GL, Zhang X, Fathman CG, Glimcher LH. A novel transcription factor, T-bet, directs Th1 lineage commitment. Cell. 2000;100(6):655-69.

Takaishi M, Ishizaki M, Suzuki K, Isobe T, Shimozato T, Sano S. Oral administration of a novel RORgammat antagonist attenuates psoriasis-like skin lesion of two independent mouse models through neutralization of IL-17. J Dermatol Sci. 2017;85(1):12-9.

Tan KW, Griffiths CE. Novel systemic therapies for the treatment of psoriasis. Expert Opinion Pharmacother. 2016;17(1):79-92.

Tsai TF, Ho JC, Song M, Szapary P, Guzzo C, Shen YK, et al. Efficacy and safety of ustekinumab for the treatment of moderate-to-severe psoriasis: a phase III, randomized, placebocontrolled trial in Taiwanese and Korean patients (PEARL). J Dermatol Sci. 2011;63(3):154-63.

Williams LM, Rudensky AY. Maintenance of the Foxp3dependent developmental program in mature regulatory $\mathrm{T}$ cells requires continued expression of Foxp3. Nat Immunol. 2007;8(3):277-84.

Yang J, Sundrud MS, Skepner J, Yamagata T. Targeting Th17 cells in autoimmune diseases. Trends Pharmacol Sci. 2014;35(10):493-500.

Yawalkar N, Tscharner GG, Hunger RE, Hassan AS. Increased expression of IL-12p70 and IL-23 by multiple dendritic cell and macrophage subsets in plaque psoriasis. J Dermatol Sci. 2009;54(2):99-105.

Yu SF, Zhang YN, Yang BY, Wu CY. Human memory, but not naive, CD4+ T cells expressing transcription factor T-bet might drive rapid cytokine production. J Biol Chem. 2014;289(51):35561-9.

Zhang Y, Luo XY, Wu DH, Xu Y. ROR nuclear receptors: structures, related diseases, and drug discovery. Acta Pharmacol Sinica. 2015;36(1):71-87. 
Zhu K, Ye J, Wu M, Cheng H. Expression of Th1 and Th2 cytokine-associated transcription factors, T-bet and GATA-3, in peripheral blood mononuclear cells and skin lesions of patients with psoriasis vulgaris. Arch Dermatol Res. 2010;302(7):51723.

Received for publication on $25^{\text {th }}$ September 2017 Accepted for publication on $09^{\text {th }}$ April 2018 